\title{
Archéopages
}

Archéopages

Archéologie et société

Hors-série 3 | 2012

Nouveaux champs de la recherche archéologique

\section{L'archéologie préventive : un outil pour la construction de l'aménagement du territoire}

Marie-Odile Lavendhomme

\section{OpenEdition}

1 Journals

Édition électronique

URL : https://journals.openedition.org/archeopages/464

DOI : 10.4000/archeopages.464

ISSN : 2269-9872

Éditeur

INRAP - Institut national de recherches archéologiques préventives

Édition imprimée

Date de publication : 1 janvier 2012

Pagination : 51-55

ISSN : 1622-8545

Référence électronique

Marie-Odile Lavendhomme, "L'archéologie préventive : un outil pour la construction de

l'aménagement du territoire », Archéopages [En ligne], Hors-série 3 | 2012, mis en ligne le 01 janvier

2012, consulté le 23 février 2023. URL : http://journals.openedition.org/archeopages/464 ; DOI :

https://doi.org/10.4000/archeopages.464 


\section{L'archéologie préventive : un outil pour la construction de l'aménagement du territoire}

Marie-Odile Lavendhomme

Inrap

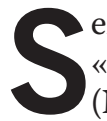
elon Pierre Nora, les lieux de mémoire sont « des rituels d'une société sans rituel » (Nora, 1997). Autrement dit, notre civilisation a besoin d'un signal avec toute sa dimension historique pour donner du sens à notre territoire. Nous avons besoin $\mathrm{du}$ " monument historique » au sens donné par Françoise Choay dans son Allégorie du patrimoine (Choay, 1999). Les métropoles actuelles sont de plus en plus homogènes et à la recherche de sens. Cette recherche identitaire, ce travail sur la réinvention du patrimoine, est œuvre quotidienne de l'urbaniste (Bourdin, 1984).

L'archéologie a pour objectif de participer à l'écriture de l'histoire de l'humanité à travers la détermination, la caractérisation et l'interprétation des vestiges matériels mis au jour, tant par l'étude du bâti que par les fouilles. Par un regard nouveau, l'archéologie, et plus singulièrement l'archéologie préventive, peut apporter une connaissance approfondie du territoire (Lavendhomme, À paraître). Le croisement de réflexions patrimoniales, historiques et urbaines permet de mieux appréhender la place de vestiges archéologiques dans les métropoles actuelles. Leur découverte pose inévitablement la question du sens du monument dans la ville, la question des lieux de mémoire.

Des recherches archéologiques liées à l'aménagement du territoire. Les travaux d'aménagement du territoire entraînent la destruction irréversible de sites archéologiques sur des milliers d'hectares. A contrario, avec ses quelques 10 ooo hectares sondés et 200 à 300 fouilles annuelles, l'Inrap contribue fortement à l'accroissement des données scientifiques et de la connaissance. Les nombreuses publications issues des découvertes effectuées, démontrent l'évolution de la recherche en ce domaine. Les méthodes liées à l'archéologie préventive permettent d'appréhender des espaces nettement plus importants dans une optique dynamique. Nous sommes passés, en moins d'un demi-siècle, d'une archéologie centrée sur le monument, aux réflexions sur le territoire en mutation.

Le «monument archéologique», au sens large du terme, est toujours au cœur de nos préoccupations, comme le démontrent quelques unes des découvertes remarquables puisées dans les rapports d'activité de l'Inrap (Rapport d'activité, 2004 à 2010) : une quinzaine unités d'occupations paléolithiques et mésolithiques à Mareuil-sur-Cher (Loir-et-Cher), un monument mégalithique à Belz (Morbihan), une enceinte du Néolithique à Pont-sur-Seine (Aube), les premiers bâtiments de la cité phocéenne archaïque à Marseille, un riche sanctuaire gaulois à Bessines (Deux-Sèvres), deux thermes antiques juxtaposés à Belley (Ain), un édifice de spectacle antique et l'aula d'un palais épiscopal à Valence, des fours à chaux à Nespouls (Corrèze), les baraquements d'un camp napoléonien à Étaplessur-Mer (Pas-de-Calais), une des plus anciennes habitations-sucreries de Guadeloupe...

Ces analyses monumentales servent de base aux réflexions territoriales. Ainsi, les questionnements actuels sur l'archéologie de la ville tournent autour du système de mise en place des principales aires urbaines actuelles. L'analyse des processus de mutations s'appuient sur la description des périodes charnières dans l'évolution des villes, la fin de l'indépendance gauloise et les évolutions entre l'agglomération gauloise et l'urbanisation romaine, l'Antiquité tardive et la lente mise en place des villes médiévales, la fin du Moyen Âge et le développement des villes modernes. Trois grandes évolutions de la ville sont attestées avec la reconstruction de la ville sur elle-même par lente assimilation, l'évolution radicale par le système de la table rase et enfin l'évolution via un déplacement de la ville sur un même territoire. La part des facteurs géomorphologiques est confrontée à celle des facteurs culturels : décision politique interne ou externe, mise à l'écart des principaux réseaux d'échange économique... Ces différentes évolutions des villes sont liées à des facteurs physiques (des espaces plus ou moins propices aux installations humaines à un instant donné, des facteurs politiques (des territoires de décisions locaux ou externes, des lieux plus ou moins stratégiques), des facteurs économiques (proximités de voies de communications)...

Face aux connaissances archéologiques renouvelées, plusieurs perspectives peuvent être formulées pour créer des liens entre le passé et le présent: le passé magnifié et fantasmé avec la question du patrimoine, soit remarquable, soit banal ; la connaissance du vécu et de l'intelligence du site pouvant entraîner une économie et une écologie, voire éviter une tragédie; enfin la connaissance territoriale permettant l'intégration du projet dans sa structuration diachronique. Trois lectures différentes des vestiges archéologiques (les lectures monumentale, matérielle et territoriale) permettent trois types de projets d'urbanisme: les projets de composition urbaine, d'économie de moyens et de lieux fédérateurs.

Le passé magnifié dans un projet de composition urbaine. La première piste de réflexion voit l'archéologie à travers l'analyse du monument pour le mettre dans une perspective historique. À travers celle-ci, les vestiges archéologiques deviennent des monuments historiques.

La fouille, sous la responsabilité de J. Catalo, de l'aile méridionale du tribunal de grande instance de Toulouse a permis à la maîtrise d'ouvrage, après la découverte des vestiges du château Narbonnais, d'engager des études de faisabilité (automne 2005), puis d'adaptation du projet judiciaire (Catalo, 2007; Catalo, Cazes, 2010). Les restes archéologiques sont conservés au niveau des fondations sur environ 4 mètres de hauteur. Ils sont intégrés à une perspective historique des implications politiques, sociales 


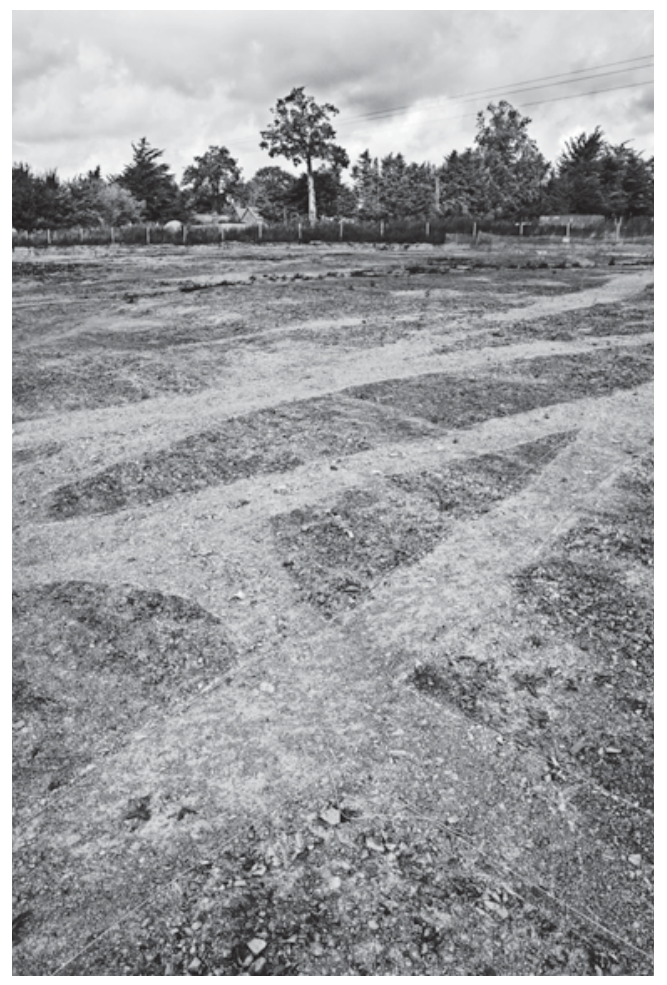

กั่

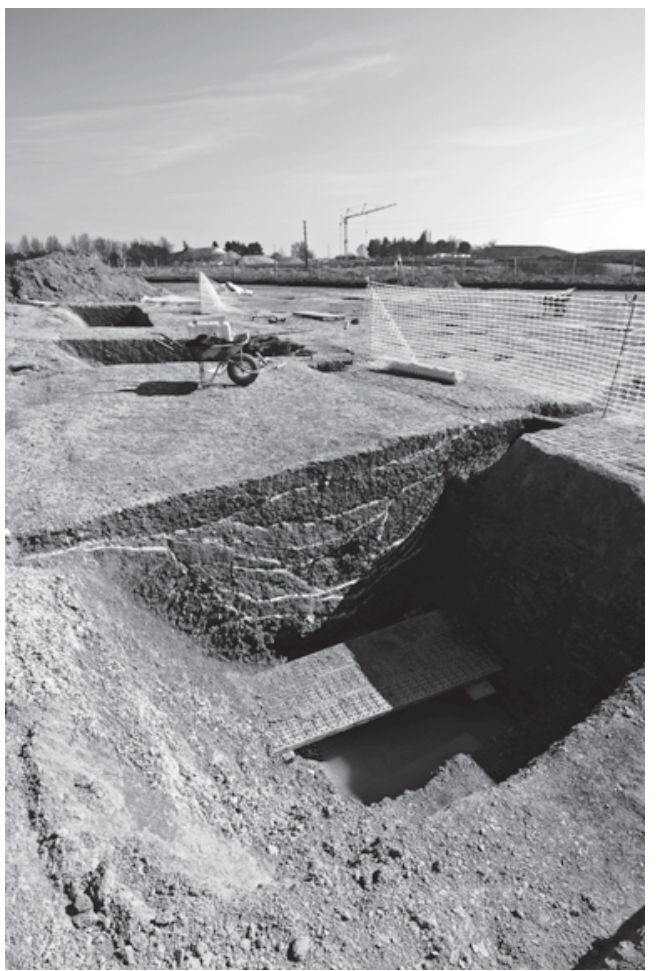

[Fig.1] Traces des structures après décapage et avant la fouille. Ici les fossés du haut Moyen Âge :

à l'intérieur des espaces circonscrits s'organisent les unités d'habitation, mais aussi les activités domestiques et artisanales.

[Fig.2] Voie en cours de dégagement.

[Fig.3] Coupe stratigraphique d'un fossé d'enclos.

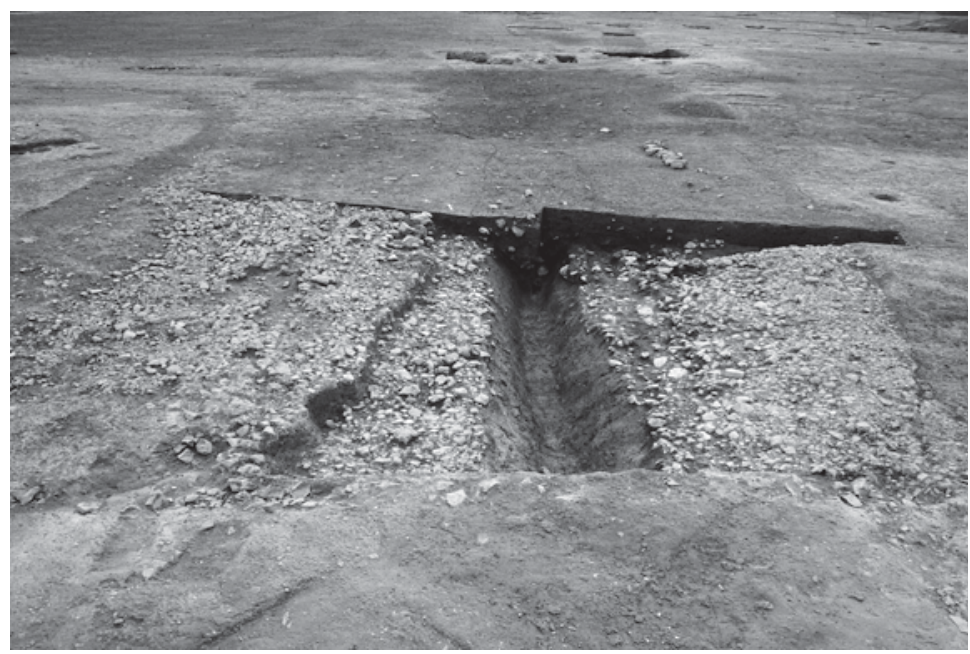

Fouille du site de la Perdriotais

à Châteaugiron (Ille-et-Vilaine) en 2008.

Comme le démontre la fouille réalisée en 2009 et 2010 par l'Inrap sous la responsabilité d'Isabelle Catteddu sur une trentaine d'hectares, le projet d'aménagement à Châteaugiron (Ille-et-Vilaine), près de Rennes, se trouve dans un espace où le paysage est façonné depuis des millénaires, les axes structurant le paysage (Cattedu, 2009, 2011, à paraître). Ainsi, les limites de certaines parcelles du cadastre actuel ou certains fossés de drainage reprennent des limites antiques ou médiévales [Fig. 1-3]. Le plan archéologique pouvait être superposé au plan d'aménagement. C'est une question de bon sens. Pourquoi, dans un espace structuré depuis des millénaires modifier les écoulements des eaux, modifier la forme des paysages alors que cette structure a apporté la preuve de son efficacité pendant des millénaires? Ainsi, le maître d'ouvrage (le Groupe Giboire et Nexity Foncier Conseil) a adapté son projet d'aménagement et repris ces éléments pérennes, tenant compte des connaissances physiques qu'ont délivrées les études archéologiques. Interrogé par l'Inrap, Guillaume Loyer, directeur de l'aménagement chez Giboire, précise que «la description des couches fouillées par l'Inrap nous a aidés à appréhender le terrain en vue de nos constructions. L'archéologue a souligné des similitudes avec nos plans. Comme nos ancêtres, nous avions intégré la topographie du terrain dans nos choix. Nos futurs cheminements correspondaient à plusieurs cheminements anciens, qui eux-mêmes avaient perduré durant les différentes occupations. De même, nos îlots d'habitation recoupaient ceux du haut Moyen Âge... Nous avons alors souhaité prendre en compte ces traces du passé». Selon le souhait de la commune, les rues et la future école seront ainsi baptisées en référence aux vestiges. 

concernés sont de hauts lieux collectifs, conservés car exceptionnels.

La mise en valeur recherche les qualités esthétiques et formelles des vestiges. Ils s'intègrent visuellement à leur environnement par une enveloppe architecturale adéquate. La valeur historique s'avère fondamentale : il s'agit de montrer l'évolution d'un site en expliquant ses différentes phases de construction. Cette démarche éducative permet à la population de voir son histoire, de comprendre son passé, son origine. Un site ne peut être bien mis en valeur que s'il est compris, donc étudié. Les connaissances historiques liées à une esthétique irréprochable font la force des propositions architecturales et urbaines. Sur les multiples opérations préventives suivies de recherches, seuls quelques vestiges exceptionnels sont intégrés dans les projets d'aménagement, les autres étant détruits.

L'intelligence du site retranscrite dans un projet d'économie de moyens. Dans la deuxième proposition, les artefacts et « écofacts » découverts par les archéologues sont réinterprétés par l'urbaniste dans son projet. L'espace physique est compris et accepté comme une évidence dans les projets. une succession d'artefacts pouvant intégrer le projet, comme l'illustre celui de Châteaugiron [cf. encadré p. 52]. Cet exemple s'inspire de l'approche développée dans l'écologie industrielle, nouvelle pratique de management environnemental partant des différentes contraintes, cherchant à intégrer l'environnement dans sa stratégie et favorisant «le fonctionnement quasi cyclique des écosystèmes naturels» (Erkmann, 1998). Il rend compte de la problématique de l'aménagement du territoire dans une approche durable en intégrant les connaissances physiques du territoire dans le projet d'urbanisme. La valorisation des déchets comme celle des ressources, en bouclant autant que possible les cycles de matières, sont les éléments de méthode qui peuvent êtres retenus pour notre propos. Alexandre Chemetoff sur l'île de Nantes (Loire-Atlantique) utilise ce principe dans son projet: une économie de moyens permettant la construction d'espaces publics à moins de 100-120 $€ / \mathrm{m}^{2} \mathbf{1}$.

Le projet d'urbanisme utilise la même technique adoptée durant des générations : le réemploi, «l'écologie industrielle» ${ }^{2}$. Les vestiges ne sont pas dénaturés, mais utilisés pour un projet futur. Blocs

1 Il s'agit de ne pas modifier les pentes ou les descentes d'eau, de favoriser la récupération des matériaux, d'éviter les terrassements et de modifier le projet initial en s'adaptant aux découvertes afin d'en conserver le maximum d'éléments.

2 cf Archéopages 29, "Recyclage et remploi", Inrap 2010 et culturelles du lieu. Les vestiges archéologiques La fouille préventive est d'abord perçue comme

des concepts nés de l'évolution des territoires (Rémy, Voye, 1992). La fouille préventive est considérée comme l'objet d'une réflexion territoriale. Les hypothèses liées à la détermination des facteurs de mutation sont prises en compte dans le projet. Aucune ville ne revendique clairement la connaissance archéologique pour porter un projet qui n'est pas le fruit d'une décision politique volontariste. Pourtant plusieurs décisionnaires s'en inspirent de manière plus ou moins consciente comme on le voit à Bordeaux [cf. encadré p. 53].

Pour étayer ce propos, prenons comme exemples trois villes actuelles du département de la Loire ayant fait l'objet de recherches archéologiques (Lavendhomme, 1997): Saint-Étienne, Feurs et Roanne. La première est l'actuelle préfecture, située au sud du département, au débouché de la vallée du Giers, reliant la vallée du Rhône et celle de la Loire. La deuxième était capitale de cité des Ségusiaves avant de perdre, petit à petit, son importance pour devenir finalement un chef-lieu de canton. La dernière est l'une des deux souspréfectures de la Loire, au nord du département.

L'évolution d'une ville doit se comprendre dans son territoire: Roanne évolue par lente assimilation; Feurs se transforme depuis la fin de la période gauloise par le système de la table rase; Saint-Étienne se construit par déplacements successifs de la ville au sein d'un vaste territoire. Le territoire de décision est un facteur important dans ces évolutions différentes. À Roanne, la décision de poursuivre l'urbanisation fut prise localement. À Feurs, le choix de raser le village gaulois pour reconstruire une ville romanisée fut une décision romaine. Les déplacements successifs de Saint-Étienne ne peuvent s'expliquer que par l'extrême tension liée à l'emplacement stratégique de cette zone située entre Rhône et Loire et qui évolue par d'importantes volontés politiques.

Dans cet exemple, le territoire est considéré comme le produit de la relation qu'entretiennent la société et l'espace. L'espace et le temps sont considérés en ce qu'ils ont de social, donc de relatif (Rémy, Voye, 1992). Les spécificités relèvent de déterminants comme la dimension du politique, de l'économique, du religieux, du culturel. Si la relation entre le lieu d'habitat et la vie sociale change, cela entraîne des modifications de l'urbanisation de la ville. La détermination de ces mutations permet de mettre en avant des lieux fédérateurs, porteurs de projet. L'émergence de ceux-ci est liée à l'héritage local (son sens, sa culture), au respect du site (son espace physique) en tenant compte des impacts environnementaux et de sa faisabilité économique (partenariat publicprivé) (Hanin, 2004). Les traces du passé servent à créer l'avenir. Le projet est lié à l'analyse de ces empreintes ; fondé sur les lieux fédérateurs, il permet de percevoir d'abord la ville, et donc le patrimoine, comme un espace social global dans la métropolisation. Les connaissances archéologiques se trouvent ainsi mises en valeur.

Les connaissances archéologiques traduites dans un projet de lieux fédérateurs. La troisième piste de réflexion est issue d'une lecture archéologique à l'échelle territoriale, permettant une intégration médiéval bordelais ou rails des anciens chemins de fer nous rappellent l'histoire du site qui, ainsi, n'est pas oublié. La mémoire est là: par son utilisation, le «sens » est donné (Choay, 1999 ; Nora, 1997). Le patrimoine, qu'il soit exceptionnel ou banal, est considéré pour sa valeur physique. Tous les artefacts et «écofacts» intègrent potentiellement les projets qui, eux-mêmes, tiennent compte de l'intelligence du site.

\section{L'archéologie préventive : des connaissances utiles pour l'évolution des territoires? Les lieux peuvent être définis comme des faits, ayant une forme,}


Réaménagement stratégique à Bordeaux.

Les multiples opérations archéologiques concomitantes de l'Inrap, réalisées entre 2000 et 2003 (sous les responsabilités de Wandel Migeon, Christophe Sireix, Frédéric Gerber et Kristell Chuniaud), lors de la création du tramway, de la construction de parkings souterrains et du réaménagement de trois places stratégiques, prennent place dans le projet d'urbanisme [Fig.1]. Les trois places liées à ce projet sont: la place Pey-Berland, la place de la Victoire et celle de la Comédie. Formant un triangle, elles se répondent pour permettre des échanges sociaux concentrés dans le centre. Elles sont également situées à l'emplacement de lieux occupés depuis l'Antiquité, voire depuis la Protohistoire (Lavaud, 2009). La ville de Bordeaux a donc évolué dans la longue durée par le système de la lente évolution sur place à travers une assimilation successive des différentes cultures [Fig.2]. La connaissance de ce type d'évolution diachronique de la ville, dans le cadre d'une unité de lieu, permet l'appropriation de vestiges archéologiques en les incorporant dans le projet de réaménagement, voire en le modifiant: la découverte d'une tour-porche de la cathédrale Pey-Berland (Migeon, 2003), nécessitant la modification du projet, est intégrée par une plaque commémorative expliquant la découverte dans un langage architectural propre à cette place; le réaménagement du Cours du Chapeau Rouge redynamise une rue de tout temps stratégique (Chuniaud, Sireix, 2006) ; les quais retrouvent leur importance tout en se modifiant radicalement (du port d'origine antique puis médiévale, ils deviennent un haut-lieu de rencontre et de détente en étant réinterprétés comme une promenade entièrement réaménagée sur $5 \mathrm{~km}$ de long) (Gerber, 2006); la place de la Comédie redevient ce haut-lieu qu'il a toujours été, cœur du village protohistorique, emplacement d'un temple antique et maintenant centre culturel et touristique (Sireix, Boccacino, 2007). Ainsi les différentes places stratégiques actuelles se répondent, rappelant par-là que cette ville n'a pas qu'un riche passé inscrit dans une architecture du xviiie siècle, mais un encrage historique nettement plus ancien. Ces lieux sont réinvestis par la population qui désertait petit à petit le centre-ville.

Mais quel avenir aura le cœur historique face à «Euratlantique», situé plus au sud ? Cette opération d'intérêt national regroupe l'ensemble des facteurs pour qu'une mutation se produise : un territoire de décision externe (l'État); un haut-lieu collectif (gare et centre d'affaire); la proximité d'un axe de communication rapide (TGV). Ce projet national provoquera-t-il un déplacement du centre historique de Bordeaux? Une question reste en suspens: comment seront gérées sur le temps long les contraintes hydrologiques majeures de la zone?
Les berges de la Garonne réhabilitées

- Le tramway

- Les places stratégiques redynamisées

Le périmètre du secteur classé au titre du patrimoine mondial de l'UNESCO

Le secteur sauvegardé

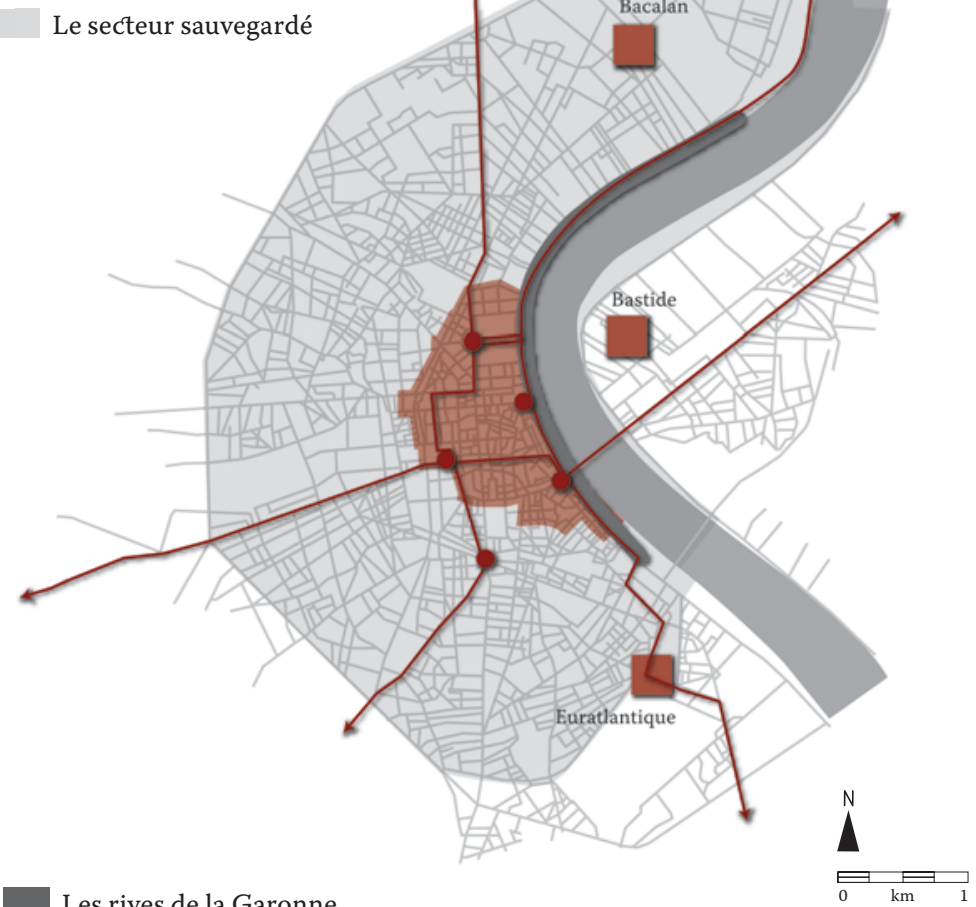
durant l'Antiquité

L'occupation dès le $\mathrm{VI}^{\mathrm{e}} \mathrm{S}$ av. notre ère

La ville augustéenne

L'extension du Haut-Empire

....... Le resserement dans le castrum de l'antiquité tardive

L'extension du XIII' $\mathrm{e}$.

---- L'extension du $\mathrm{xIV}^{\mathrm{e}} \mathrm{s}$.

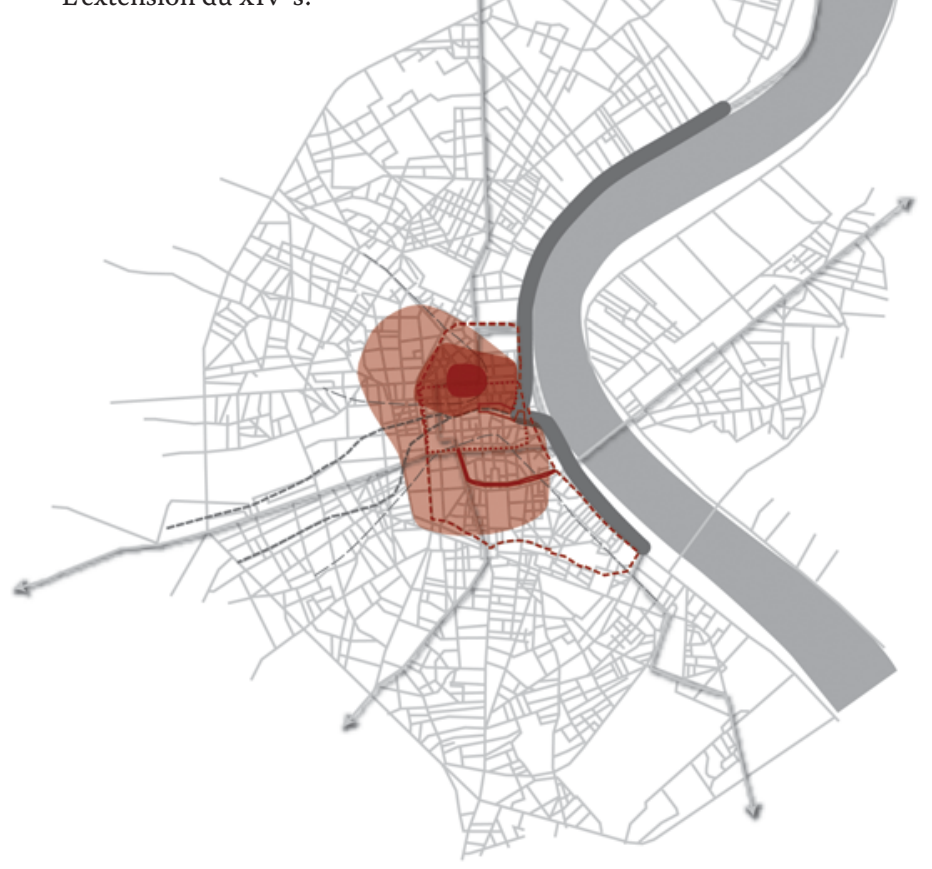

[Fig.1] Évolution de la ville de Bordeaux depuis son origine jusqu'à la période médiévale.

[Fig.2] Le projet urbanistique du centre de Bordeaux. 
une chronologie, une fonction et une valeur d'usage, de reconnaissance sociale et culturelle, ce qui les établit en force constituante.

Françoise Choay parle d'une évolution de la vision du monument, du signe au signal (Choay, 1999, p. 15). Le nom «monument » vient de monumentum qui dérive de monere (avertir, rappeler), qui interpelle la mémoire. Ce sens originel a progressivement disparu, au profit d'un monument à valeur historique, esthétique, de pouvoir ou de grandeur. De «signe», de «sens», il est devenu un «signal», une «image». Il est maintenant dépourvu de sens, de vision du temps et de durée. Prolongeant cette position, Pierre Nora parle de mémoire car elle est déchirée (Nora, 1997, p. 29). Reste néanmoins un sentiment de continuité qui passe par les lieux de mémoire. La mémoire collective, la mémoire intégrée et spontanée, la mémoire sans passé qui reconduit éternellement l'héritage, renvoyant aux ancêtres et aux mythes, est remplacée par notre mémoire qui n'est qu'histoire, trace et tri. La mémoire n'est plus « habitée », il lui faut donc des lieux. Ces lieux ou monuments se trouvent dans un espace, dans un territoire et dans une temporalité (Hanin, 2004, p. 5). L'espace est de l'ordre du donné, comme le relief ou les occupations ; il peut être quantifié et objectivé. Le territoire est l'espace maîtrisé par l'homme pour «le faire sien». Cette maîtrise physique, sociale, économique et culturelle est en perpétuelle évolution selon le projet qui s'y rapporte. Le lieu est la maîtrise de l'espace «en fonction de codes collectifs ». Les différents lieux mis en réseau constituent le territoire. Le lieu peut se charger de « valeurs », il permet alors de distinguer les lieux « ordinaires » des «hauts lieux» avec leur pouvoir fédérateur.

L'usage partagé, la reconnaissance sociale, la force constituante du lieu repose parfois sur un héritage ancestral. Et cette force fédératrice place les individus dans une vision commune dynamique. Le statut particulier du lieu est apporté par l'analyse historique montrant son importance particulière dans l'évolution de la ville. Il devient alors lieu de pouvoir ou stratégique pour le développement de la ville, haut lieu, lieu symbolique. Il oscille entre «monument, mémoire, collectif, objet, mythe, signe» et «monument historique, histoire, individu, savoir, signal, image». Cette oscillation passe par la maîtrise du lieu, des codes collectifs, du cadre social et culturel, du rôle identitaire pour l'individu ou la collectivité. Les vestiges archéologiques sont, par nature, des lieux oubliés. Il n'existe plus d'interaction entre le lieu d'habitat et la vie sociale. Le lien est rompu par le temps. Redécouvrir des espaces enfouis, en les réintégrant dans les processus d'urbanisation de la ville, est réalisable moyennant la connaissance, par la recherche scientifique sur le territoire, de sa structure intrinsèque diachronique. Les trois types d'évolution des villes sont liés aux facteurs politiques, économiques et physiques. Associées aux grands enseignements des villes antérieures et aux principaux facteurs actuels de métropolisation ${ }^{3}$, ils participent à une connaissance accrue de l'évolution de notre territoire sur le temps long et donc sur son développement durable.
Donner du sens à des projets d'urbanisme s'avère possible par la «connais-sens » du site et la «connaissance» du territoire. Les découvertes opérées lors des fouilles préventives peuvent à la fois être considérées comme des lieux dans une ville qu'il s'agit d'appréhender globalement, mais, en même temps, comme des matériaux et des indicateurs de la manière de gérer au mieux un espace dans le respect de son environnement physique. La triple approche du passé magnifié, de l'intelligence du site et de la connaissance du vécu entraîne une économie et une écologie spécifiques. La lecture monumentale produit un projet de composition urbaine proche de la notion de «monuments historiques ». La lecture matérielle donne un projet d'économie de moyens proche des notions de «monument» et de «mémoire» chères à Françoise Choay et Pierre Nora. La connaissance territoriale permet l'intégration du projet dans la structuration diachronique du site.

Ces propositions nécessitent la prise en compte de l'archéologie préventive dans la chaîne de l'aménagement du territoire. Les enjeux de mutation sur le temps long sont intégrés dans les projets stratégiques. Les résultats de l'archéologie préventive sont incorporés durant les projets opérationnels, celui d'aménagement évoluant en fonction des découvertes archéologiques. Ainsi le patrimoine, dans sa dimension archéologique, est non seulement un moteur du développement, mais aussi et surtout un fondement essentiel pour un développement territorial durable.

Bourdin A., 1984: Le patrimoine réinventé, Paris, Presses universitaires de France.

CATALO J., 2007: «Le château Narbonnais à Toulouse», Archéopages, 19, p. 40-45. CAtalo J., Cazes Q. (DIR.), 2010 : Toulouse au Moyen Âge, 1000 ans d'histoire urbaine 4.00-14.80, Portets-sur-Garonne, éd. Loubatières, 472 p.

CATteddu I., 2009: « Les occupations rurales de Châteaugiron », in Menez Y., Hinguant S., Fouilles et découvertes en Bretagne, éd. Ouest-France, Inrap, p. 106.

CAt teddu I., 2011 : « Châteaugiron, un terroir structuré », Dossiers de l'archéologie: Aux origines du Moyen Âge (V-XI' s.), à la découverte des sociétés rurales, $\mathrm{n}^{\circ} 344$, p. 13.

CATTEDdu I., à paraître : « Dynamique de l'occupation des campagnes : pratiques agro-pastorales et gestion de l'espace par les sociétés altomédiévales ; Étude de cas en Bretagne », Actes de la table ronde Des hommes aux champs, Caen, 29-3o septembre 2011.

ChOAy F., 1999 : L'allégorie du patrimoine, Paris, Seuil.

Chuniaud K., Sireix Chr., 2006 : Cours du Chapeau Rouge : Rapport de fouilles, Pessac, Inrap GSO.

ERKMANN S., 1998 : Vers une écologie industrielle, Paris, éd. Charles Léopold Mayer.

Gerber F., 2006 : Des rivages garonnais de Burdigala au Port de la Mer étude de 2000 ans de rapports entre Bordeaux et son fleuve : présentation des opérations et synthèse des principaux résultats: Rapport de fouilles, Pessac, Inrap GSO.

HANIN Y., 2004: Mutations spatiales et recompositions territoriales: les processus territoriaux dans le cas de Court-Saint-Étienne (Belgique), thèse de doctorat, Université Catholique de Louvain, Louvain-la-Neuve.

LAVAud S. (COORD.), 2009 : Atlas historique des villes de France: Bordeaux, 3 vol., Bordeaux, Ausonius, Aquitania.

Lavendhomme M.-O., 1997 : La Loire, Paris, Maison des sciences de l'homme, (coll. Carte archéologique de la Gaule, 42).

LAVENDHOMME M.-O., à paraître : « La place de l'archéologie préventive dans le projet d'urbanisme en France », Congrès Archeologia e citta : riflessione sulla valorizzazione dei siti archeologici in aree urbane, Rome, 11-12 février 2010.

Migeon W., 2003: Suivi des déviations de réseaux du Tramway de Bordeaux : Rapport de fouilles. Pessac, Inrap GSO.

Nora P. (DIR.), 1997 : Les lieux de mémoire. 3 vol., Paris, Gallimard.

RÉMY J., VOYE L., 1992 : La ville : vers une nouvelle définition? Paris, L'Harmattan.

Sireix Chr., Boccacino C., 2007 : Bordeaux, Parking du Grand Hôtel, 4 et 8 à 12 rue Mautrec : Rapport de fouilles, Pessac, Inrap GSO.
3 En particulier
la communication en compte de 